\title{
Robust Centered Element Concentric Circular Antenna Array with Low Side Lobe Using Variable Loading and Tapering Windows in the Presence of Array Imperfections
}

\author{
M. F. Reza, M. S. Hossain, and M. M. Rashid \\ Department of Electrical and Electronic Engineering, Rajshahi University of Engineering and Technology, Rajshahi, Bangladesh \\ Correspondence should be addressed to M. F. Reza; farhamdur@gmail.com
}

Received 8 June 2017; Accepted 25 July 2017; Published 10 September 2017

Academic Editor: Ikmo Park

Copyright (C) 2017 M. F. Reza et al. This is an open access article distributed under the Creative Commons Attribution License, which permits unrestricted use, distribution, and reproduction in any medium, provided the original work is properly cited.

\begin{abstract}
This paper presents centered element concentric circular antenna array (CECCAA) using variable diagonal loading (VDL) technique and different filtering windows. The different filtering windows are modified to apply in the CECCAA system. The modified novel technique not only is able to reduce the side lobe level (SLL) but also has the ability to detect and highly attenuate the directional interferences. However, the performance of CECCAA system is degraded in the presence of array imperfections. This performance degradation problem due to array imperfections can be improved by using robust techniques. The proposed technique is also robust against array imperfections and improves the performance. Moreover, the performance of the proposed structure is better than a concentric circular antenna array (CCAA). Several examples are presented to analyze the performance of proposed beamformer by using different tapering windows.
\end{abstract}

\section{Introduction}

The technique that combines signals from array elements of an array antenna system after multiplying signal from each antenna element with a specified weight to steer the beam towards the desired direction is called beamforming $[1,2]$. A concentric circular antenna array (CCAA) consists of more than one circular antenna array and all the ring antenna arrays having different radius share a common center. A CCAA structure can be converted into the proposed centered element concentric circular antenna array (CECCAA) structure by simply using an extra antenna element at the center of the structure. The entire space can be covered and the main lobe can be oriented in any desired direction by using CECCAA beamformer. Using same space, a large number of antenna elements can be used in a CECCAA system in comparison to a uniform circular array (UCA) system, whereas it is difficult to install UCA system with large number of antenna elements because of its size limitation [2]. As the CCAA processor offers better performance than the existing UCA processor [3], a CECCAA structure is chosen in this paper.
The algorithm called cat swarm optimization (CSO) is presented in [4] to reduce the side lobe level (SLL) and to improve the directivity of a CCAA system with isotropic elements. To optimize the radius of the ring and the interring spacing of a CCAA system with both reduced SLL and reduced number of antenna elements, adaptive techniques are discussed in [5-7]. A hybrid method based on convex optimization and a deterministic approach to optimize both SSL and first null beamwidth (FNBW) for sparse concentric ring arrays is addressed in [8]. The direction of arrival (DOA) estimation technique in the presence of high and low noisy environment for both circular and concentric circular antenna arrays is discussed in $[9,10]$.

A popular array geometry is CCCA in which the rings as well as the individual ring elements are separated by almost half of the wavelength. That means the distance between the elements of a ring should be half-wavelength and the interring spacing also should be half-wavelength $[11,12]$. This geometry can be obtained, if the number of elements of the neighbored rings is incremented by 6 elements [12]. Consequently, the rings will be separated by the nearest distance to a half-wavelength (about 0.4775 of the wavelength) [6]. 
The proposed CECCAA beamformer with half-wavelength interelement spacing can be obtained by using 6 elements at the first ring and number of elements of the neighbored ring is incremented by 6 elements and interring separation is about 0.4775 times of the wavelength. The CECCAA at this separating distance will have the optimum radiation pattern and desirable side lobes locations but still has higher side lobes levels which are not appropriate in many applications demanding lower side lobes. To reduce the SLL of the CCAA system, different tapering windows such as Triangular, Hamming, Hanning, Blackman, and Binomial windows are modified and used in [6]. However, the conventional tapered CCAA beamformers discussed in [6] are able to reduce SLL only and are not able to attenuate directional interferences.

This paper proposes tapered minimum variance distortionless response (MVDR) CECCAA beamformers by using different windows, which have the ability to attenuate directional interferences. The performance of a MVDR beamformer degrades if there is any difference between steering direction and actual signal direction [13-18]. To improve the performance of the beamformer, different loading techniques are used in [19-25]. This paper also proposes a novel technique named tapered robust MVDR CECCAA beamformer. The proposed beamformer not only is able to reduce the SLL but also attenuates the directional interferences and improves performance in the presence of look direction disparity by using different modified filtering windows and variable diagonal loading (VDL) technique.

The proposed beamformer has the following desirable properties:

(i) The main beam can be steered at any arbitrary direction by using CECCAA beamformer.

(ii) CECCAA offers better performance than existing CCAA beamformer.

(iii) The proposed beamformer by using different modified filtering windows provides lower SLL.

(iv) The proposed processor is also robust against look direction mismatch.

(v) It provides the ability to cancel directional interferences.

This paper is divided into six sections. Section 2 introduces the array geometries and signal model used in this work. Section 3 discusses ring coefficient by using different windows. Section 4 proposes a novel tapered robust beamforming technique. Section 5 analyzes and discusses the simulation results, and, finally, Section 6 concludes the paper.

\section{System Model}

In many applications, it is essential to scan the object between $0^{\circ}$ and $360^{\circ}$. However, linear antenna array is able to scan the object between $0^{\circ}$ and $180^{\circ}$. CECCAA beamformer is able to cover the entire space because of its circular structure. The block diagram of a communication system using antenna array is depicted in Figure 1. After receiving the signal, signal of each antenna element is multiplied by adjustable weight

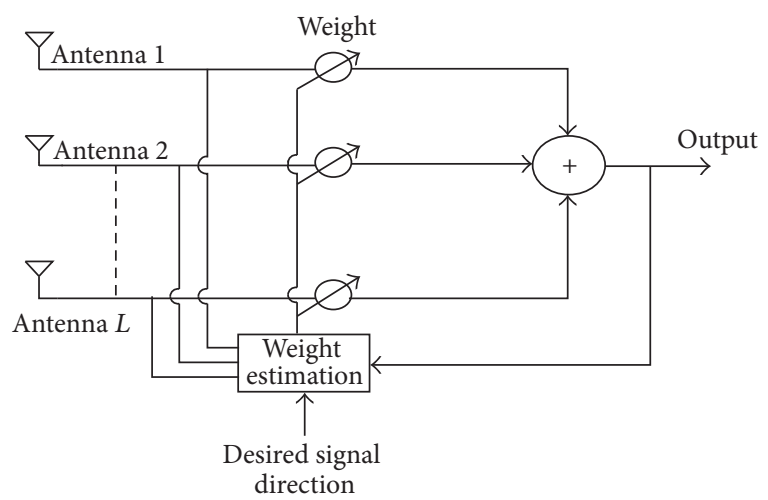

FIGURE 1: Block diagram of a communication system using an antenna array [1].

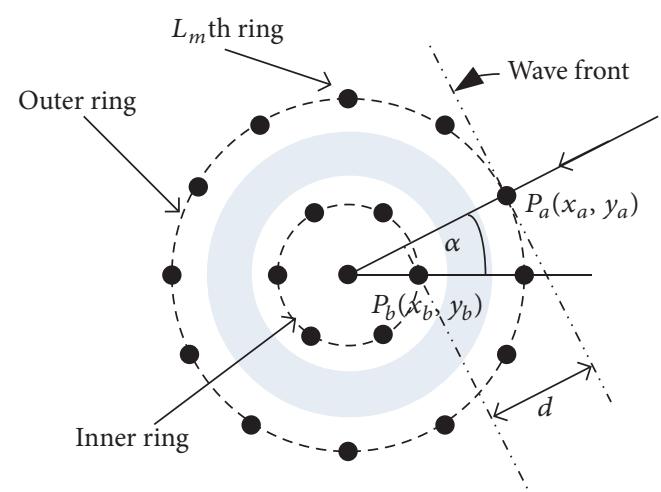

FIgURE 2: Geometry of CECCAA system.

and weights are calculated according to the direction at which one wants to receive the desired signal.

The geometry of CECCAA is displayed in Figure 2. A CECCAA beamformer consists of more than one uniform circular antenna array with an element in the center. All the ring arrays in a CECCAA system share a common center. The antenna elements are uniformly distributed, either equal or unequal in number. Referring to Figure 2, the CECCAA geometry comprises $M$ circular ring. The $m$ th ring consists of $L_{m}$ isotropic elements equally spaced with the center at the origin and $r_{m}$ is the radius of the ring, where $m=1,2,3, \ldots, M$. The elements in each ring are assumed to be omnidirectional and the interelement spacing is kept approximately half-wavelength. The CECCAA geometry with interelement spacing approximately half-wavelength can be obtained if the number of elements in the rings is incremented by 6 or $N_{m+1}=N_{m}+6$ and interring spacing is kept approximately 0.4775 times of the wavelength [12]. In Figure 2, the dashed double-dotted line signifies a plane wave front incident on the array at an angle $\alpha$. Let us consider that the two elements of the geometry are positioned at $P_{a}$ and $P_{b}$. The time required for the wave front to arrive from point $P_{a}$ to $P_{b}$ is given by

$$
\tau=\frac{\left(\left(x_{a}-x_{b}\right) \cos (\alpha)+\left(y_{a}-y_{b}\right) \cos (\alpha)\right)}{c},
$$

where $c$ is the velocity of the signal to be received. 
The induced signal on any array elements due to $k$ th source at any time instant is articulated in complex representation as $m_{k}(t) e^{j 2 \pi f t}$. The signal from the $k$ th source is assumed to be arrived at an angle $\left(\varphi_{k}, \theta_{k}\right)$. If the time needed to induce the signal in the $l$ th element is $\tau_{l}\left(\varphi_{k}, \theta_{k}\right)$, then induced signal on any array elements can be expressed as $m_{k}(t) e^{j 2 \pi f\left(t-\tau_{l}\left(\varphi_{k}, \theta_{k}\right)\right)} \cdot m_{k}$ and $f$ represent the complex modulation frequency and carrier frequency, respectively. With $N$ directional sources and in the presence of background noise, signal induced in the $l$ th element can be expressed as

$$
x_{l}(t)=\sum_{k=1}^{N} m_{k}(t) e^{j 2 \pi f\left(t-\tau_{l}\left(\varphi_{k}, \theta_{k}\right)\right)}+n_{l}(t),
$$

where $n_{l}$ is the noise component at the $l$ th element. It is supposed that the noise is uncorrelated with directional sources; that is, $E\left[m_{k}(t) n_{l}(t)\right]=0$. Noise on different elements is assumed to be uncorrelated; that is, $E\left[n_{k}(t) n_{l}(t)\right]=0$ for $k \neq l$ and $E\left[n_{k}(t) n_{l}(t)\right]=\sigma^{2}$ for $k=l$, where $\sigma^{2}$ denotes the noise power.

\section{Tapered CECCAA Beamformer}

Conventional windows like Triangular, Hamming, Hanning, Blackman, and Binomial windows that can be modified and applied for amplitude tapering on a CCAA system to reduce SLL of the output power pattern are discussed in [6]. Each array element of a conventional linear tapered array uses a specified tapering coefficient. However, we consider the individual ring to be equivalent to an element of the onedimensional linear array in case of CCAA. So, equal weights are used by the elements of a particular ring in CCAA beamformer. As an element is used in the center of the CECCAA beamformer, $M+1$ weights are required in a $M$ ring CECCAA beamformer. The ring coefficient by using different windows for CECCAA system is given below.

In a uniformly fed CECCAA system, the coefficients of all rings are the same and they are given as

$$
\alpha_{m}=1, \quad m=0,1,2, \ldots, M
$$

For Triangular amplitude tapering in a CECCAA system of $M$ rings, the ring coefficients can be expressed as

$$
\alpha_{m}=\frac{(M-m+1)}{M+1}, \quad m=0,1,2, \ldots, M,
$$

where the coefficient for element in the center is $\alpha_{0}=1$ and coefficient for outermost ring is $\alpha_{M}=1 /(M+1)$.

The ring coefficient by using Hamming window for a $M$ ring CECCAA system can be modified as

$$
\begin{aligned}
\alpha_{m}=0.54-0.46 \cos \left(\frac{\pi(M-m-2)}{M+2}\right) & \\
m & =0,1,2, \ldots, M .
\end{aligned}
$$

Similarly, Hanning window provides ring coefficient for a $M$-ring CECCAA system as

$$
\begin{aligned}
\alpha_{m}=0.5-0.5 \cos \left(\frac{\pi(M-m-2)}{M+2}\right), & \\
& m=0,1,2, \ldots, M,
\end{aligned}
$$

where total number of rings is denoted by $M$ and ring number is denoted by $m$. For the element in the center, $m=0$; for innermost ring, $m=1$, and, for outermost ring, $m=M$.

\section{Proposed Tapered Robust Beamformer}

The tapered beamformers discussed in previous section using different windows are able to reduce SLL level but are not able to detect and attenuate directional interferences. To detect and attenuate the directional interferences, MVDR beamformer is used. However, performance of a MVDR CECCAA beamformer is degraded in the presence of look direction error. Moreover, the conventional MVDR beamformer has higher SLL level. So, a novel tapered robust MVDR beamformer, based on different modified filtering windows and robust VDL technique, is proposed in this paper. The steering vector for the proposed beamformer with $M$ circular ring and an element in the center is modified as

$$
\begin{array}{r}
\mathbf{S}_{N 0}=\left[a_{0} \mathbf{S}_{0}, a_{1} \mathbf{S}_{1}, a_{2} \mathbf{S}_{2}, \ldots, a_{m} \mathbf{S}_{m}, \ldots, a_{M} \mathbf{S}_{M}\right], \\
m=0,1,2, \ldots, M,
\end{array}
$$

where $a_{m}$ is the coefficient for $m$ th ring obtained from modified filtering windows discussed in previous section; $\mathbf{S}_{m}$ denotes the steering vector of the $m$ th ring of the circular antenna and it can be expressed as

$$
\mathbf{S}_{m}=\left[e^{j 2 \pi f \tau_{m 1}}, e^{j 2 \pi f \tau_{m 2}}, \ldots, e^{j 2 \pi f \tau_{m L_{m}}}\right],
$$

where $L_{m}$ is the total number of antenna elements of the $m$ th circular ring.

In the presence of noise and interference, the array correlation matrix of the tapered MVDR beamformer can be given as [1]

$$
\mathbf{R}=p_{s} \mathbf{S}_{\mathrm{N} 0} \mathbf{S}_{N 0}^{H}+p_{I} \mathbf{S}_{I} \mathbf{S}_{I}^{H}+\sigma_{n}^{2} \mathbf{I},
$$

where $p_{s}, p_{I}$, and $\sigma_{n}^{2}$ symbolize the desired signal power, interference signal power, and random noise power, respectively. $\mathbf{S}_{N 0}$ and $\mathbf{S}_{I}$ are the modified steering vectors at the look direction and interference direction, respectively.

To make the system robust against look direction error, diagonal loading technique is used with the proposed tapered MVDR beamformer. The new array correlation matrix due to array imperfections by using VDL technique can be calculated as [17]

$$
\mathbf{R}_{\text {new }}=\mathbf{R}+\mathbf{R}^{-1} \lambda * \mathbf{I},
$$

where $\lambda$ is expressed as

$$
\lambda=\frac{\varepsilon\left(\sigma_{n}^{2}+p_{s}\left\|\mathrm{~S}_{N 0}\right\|^{2}\right)}{\left\|\mathbf{S}_{a c}\right\|-\varepsilon},
$$


with $p_{s}$ denoting the signal power and $\sigma_{n}^{2}$ denoting the noise power; norms of the steering vectors without array imperfections and with array imperfections are denoted by $\left\|\mathbf{S}_{N 0}\right\|$ and $\left\|\mathbf{S}_{a c}\right\|$, respectively. The steering vector distortion bound $\varepsilon$ is given by

$$
\varepsilon=\max \left(\left\|\mathbf{S}_{N 0}-\mathbf{S}_{a c}\right\|\right) .
$$

After calculating steering vector $\mathbf{S}_{N 0}$ and correlation matrix $\mathbf{R}_{\text {new }}$, the weighting vector for the proposed beamformer can be calculated as

$$
W=\frac{\mathbf{R}_{\text {new }}^{-1} \mathbf{S}_{N 0}}{\mathbf{S}_{N 0}^{H} \mathbf{R}_{\text {new }}^{-1} \mathbf{S}_{N 0}} .
$$

\section{Performance Evaluation}

In this section, the performance of CECCAA system is evaluated. For analyzing the performance, a CECCAA system with 6 antenna elements in the innermost ring is considered. The interelement spacing within a ring is considered as halfwavelength and ring separation is considered as 0.4775 times of wavelength. The directional interferences are assumed to be uncorrelated with the look direction signal. The signal frequency is taken as $300 \mathrm{MHz}$.

The directivity comparison between the conventional CCAA and CECCAA beamformers is shown in Figure 3. It is observed from Figure 3 that the directivity gain of the CECCAA beamformer is $14.62 \mathrm{~dB}$, whereas, without using center element, the directivity gain is $14.43 \mathrm{~dB}$, which is lower compared to using center element. Moreover, SLL is much lower in CECCAA beamformer in comparison to the conventional CCAA beamformer. Figure 4 depicts the variation of SLL with the variation of the number of rings in the CECCAA structure. From Figure 4, one can observe how SLL varies with the variation of the number of rings and it is also observed that the SLL of the CECCAA beamformer is much lower than the CCAA beamformer.

Figure 5 depicts the comparison of the different types of CECCAA beamformers by using different tapering techniques described in Section 3. In the above-mentioned figure, the black curve is for conventional (uniformly tapered) beamformer; on the other hand, the red and blue curves are for the CECCAA beamformer with Triangular amplitude tapering and Hamming amplitude tapering, respectively. From Figure 5, it is observed that the SLL is reduced by using different tapering windows in comparison to the conventional CECCAA beamformer.

Figure 6 represents the SLL comparison of tapered beamformers using different tapering windows with the variation of the number of rings in the CECCAA structure. For a 10-ring CECCAA structure, though the SLL of a uniformly tapered conventional beamformer is $-8.676 \mathrm{~dB}$, it is reduced to $-15.97 \mathrm{~dB},-13.51 \mathrm{~dB}$, and $-13.91 \mathrm{~dB}$ by using Triangular, Hamming, and Hanning tapered CECCAA beamformers, respectively. It is also observed that, with the increase of the number of rings in a CECCAA structure, the SLL of the output power pattern is reduced and CECCAA beamformer using different tapering windows shows better performance

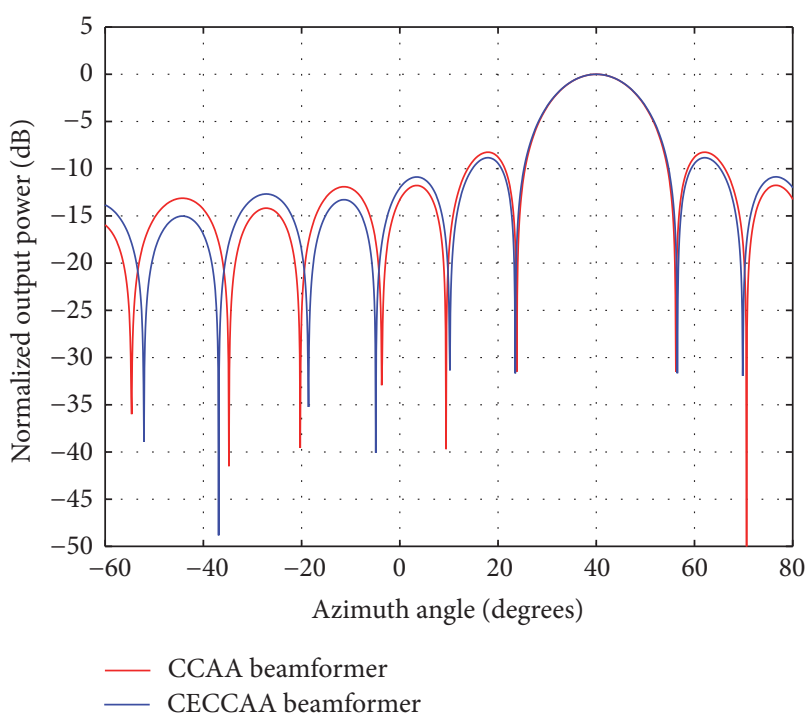

FIGURE 3: Directivity pattern comparison between the CCAA beamformer and CECCAA beamformer.

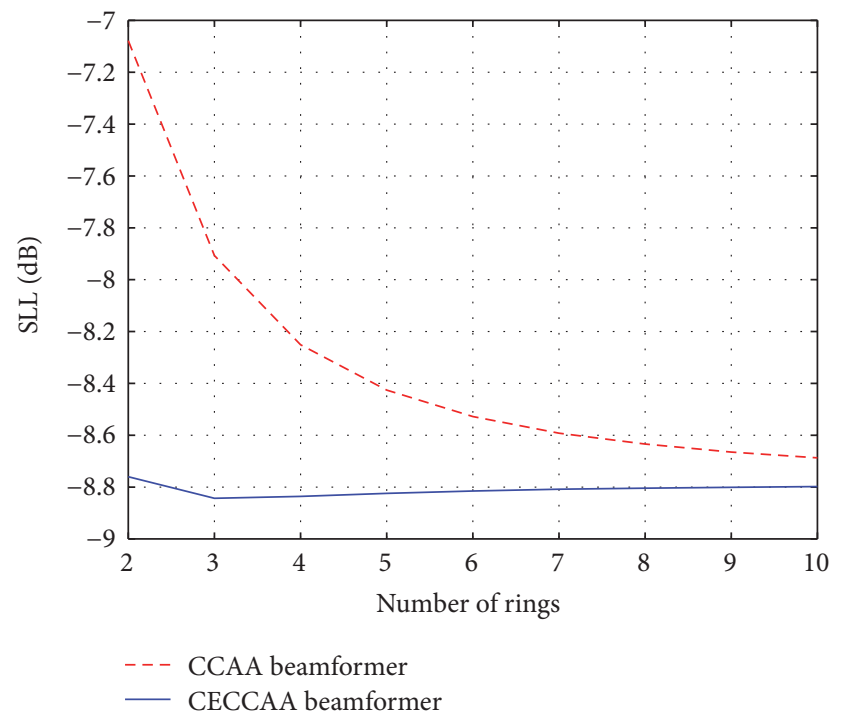

FIGURE 4: Variation of the SLL between the CCAA beamformer and CECCAA beamformer with the variation of the number of rings.

in comparison to the uniformly tapered CECCAA beamformer.

The normalized power pattern of the Triangular tapered CECCAA beamformer by using different beamforming techniques is shown in Figure 7 . The steering direction at $40^{\circ}$ and directional interferences at an angle of $-40^{\circ}$ and $-20^{\circ}$ are considered to depict those curves. Figure 7(a) represents the normalized power pattern of Triangular tapered CECCAA beamformer by using conventional technique. From this figure, it is observed that although the directional interferences are considered at $-40^{\circ}$ and $-20^{\circ}$, the Triangular tapered beamformer by using conventional technique has no ability to detect directional interferences and attenuate the interferences. It only has the ability to reduce SLL compared 


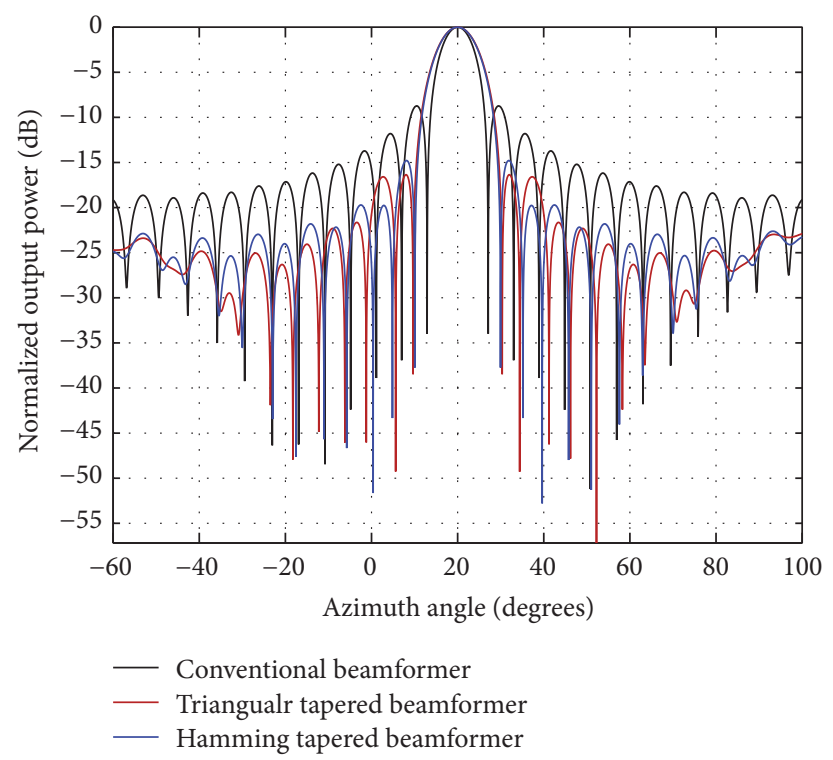

FIGURE 5: Normalized power pattern comparison of conventional CECCAA beamformer with tapered CECCAA beamformers.

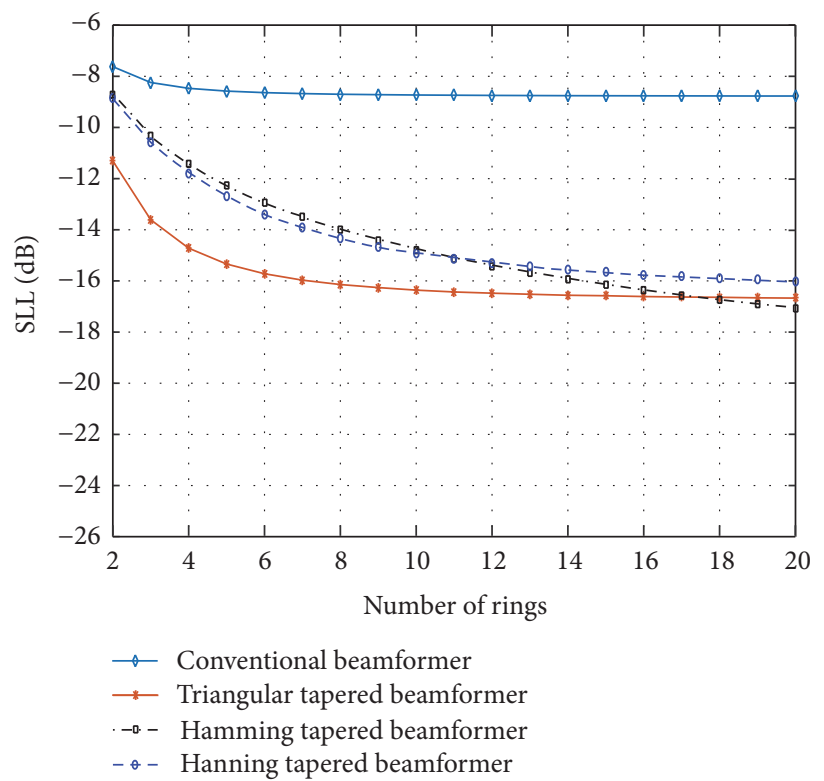

FIGURE 6: Side lobe level comparison of tapered beamformers using different windows with the variation of the number of rings.

to the uniformly tapered beamformer. Normalized power pattern of Triangular tapered MVDR CECCAA beamformer without presence of array imperfections is depicted in Figure 7 (b). From this figure, one can observe that Triangular tapered MVDR CECCAA beamformer is able to detect and attenuate directional interferences that are considered at $-40^{\circ}$ and $-20^{\circ}$ and also has the ability to reduce SLL in comparison to uniformly tapered beamformer. However, the performance of the system is degraded in the presence of array imperfections. The performance degradation problem of Triangular tapered MVDR CECCAA beamformer is shown in Figure 7(c). For depicting the figure, the original signal is considered at $45^{\circ}$. It is observed that there is high attenuation at the actual signal direction due to mismatch between steering direction and original signal direction. This performance degradation problem due to array imperfections can be minimized using the proposed tapered robust MVDR CECCAA beamformers. Figure 7(d) represents the comparison of normalized power pattern of MVDR CCAA and proposed Triangular tapered robust MVDR CECCAA by using modified triangular window and VDL technique in the presence of array imperfections. From this figure, one can 


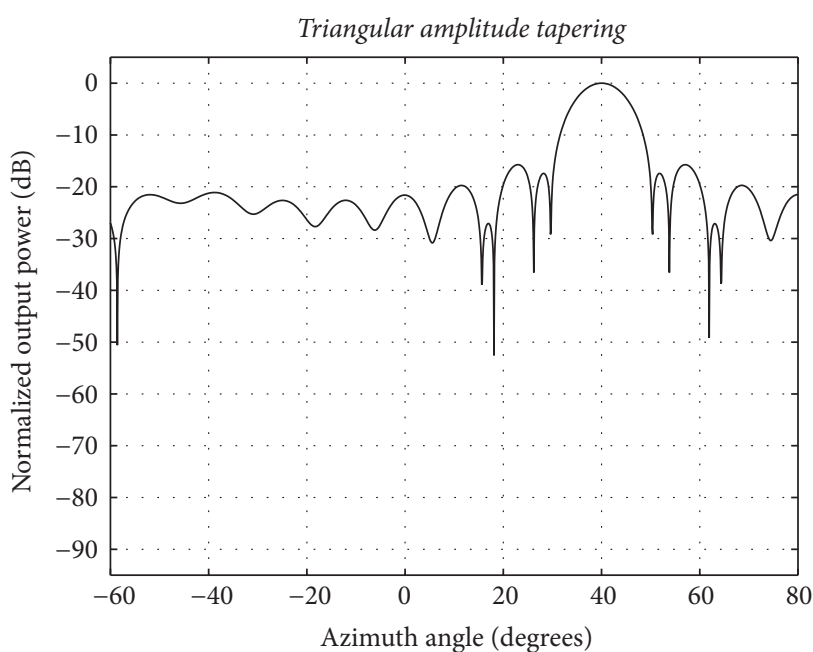

(a)

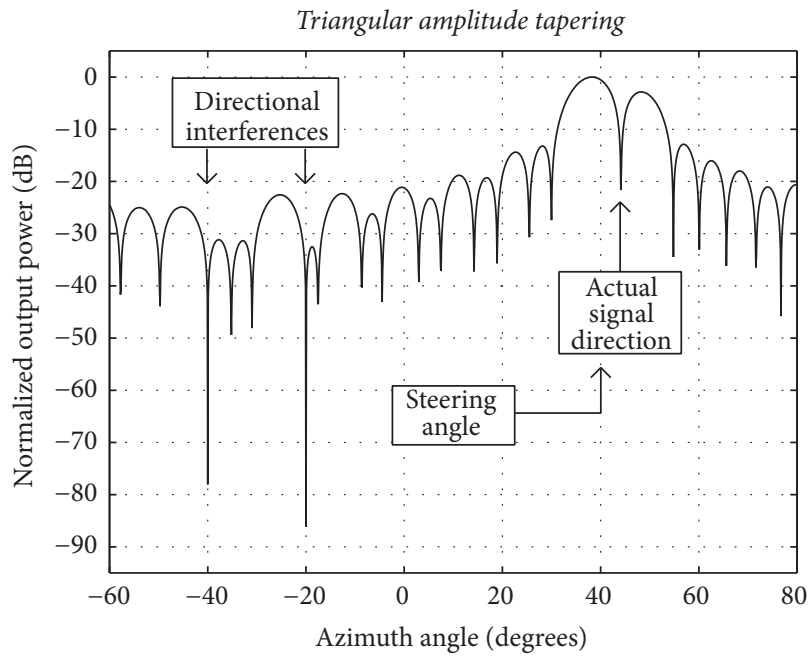

(c)

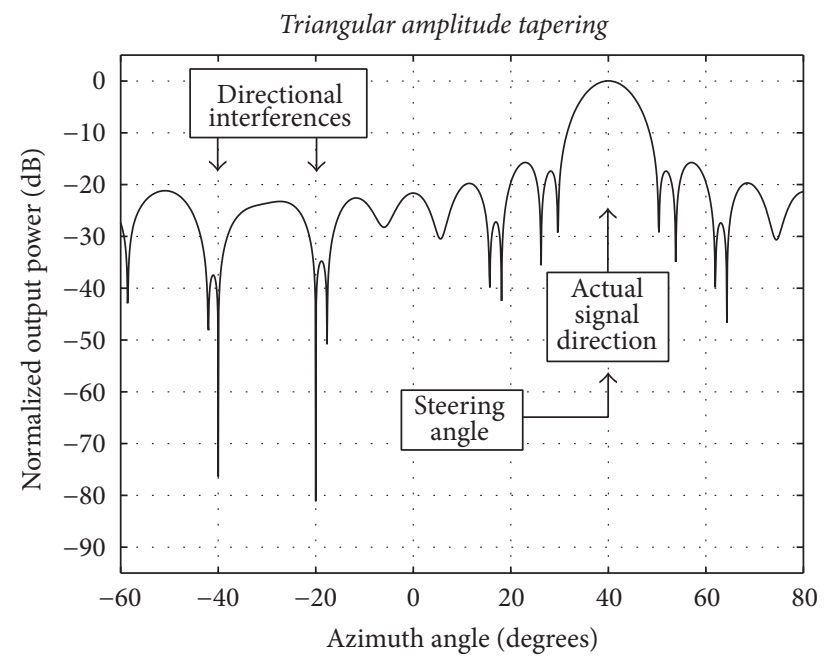

(b)

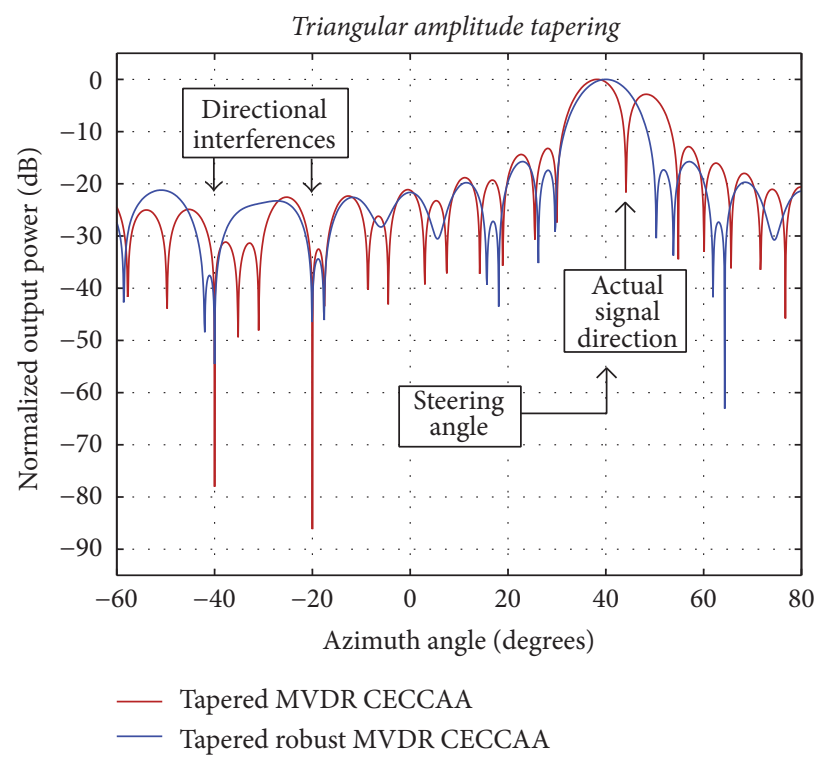

(d)

FIgURE 7: (a) Normalized power pattern of Triangular tapered CECCAA. (b) Normalized power pattern of Triangular tapered MVDR CECCAA without presence of array imperfections. (c) Normalized power pattern of Triangular tapered MVDR CECCAA in the presence of array imperfections. (d) Normalized power pattern comparison of the Triangular tapered MVDR CECCAA and proposed Triangular tapered robust MVDR CECCAA in the presence of array imperfections.

observe that the proposed beamformer not only is able to reduce SLL and attenuate directional interferences but also is robust against look direction error.

Figure 8 displays the comparison of the proposed beamformers by using Hamming and Hanning windows with the uniformly tapered beamformer. The steering direction is assumed at $40^{\circ}$ and two directional interferences are considered at $-40^{\circ}$ and $-20^{\circ}$. Figure $8(\mathrm{a})$ represents normalized power pattern of MVDR and robust MVDR CECCAA beamformer in the presence of array imperfections by using uniform tapering. On the other hand, Figures 8(b) and 8(c) represent the normalized power patterns of tapered MVDR and proposed tapered robust MVDR CECCAA beamformers in the presence of array imperfections by using Hamming window and Hanning window, respectively. All the robust beamformers are plotted by using VDL technique. From those figures, it is observed that the different tapering exhibits different power pattern and by using different filtering windows the SLL is decreased in comparison to the beamformer using uniform tapering. It is also observed that the proposed tapered beamformers, by using different tapering windows and robust VDL technique, are able to attenuate directional interference and improve the performance in the presence of look direction disparity.

Figure 9 represents 3D normalized output power pattern of a 10-ring proposed tapered robust MVDR beamformer 


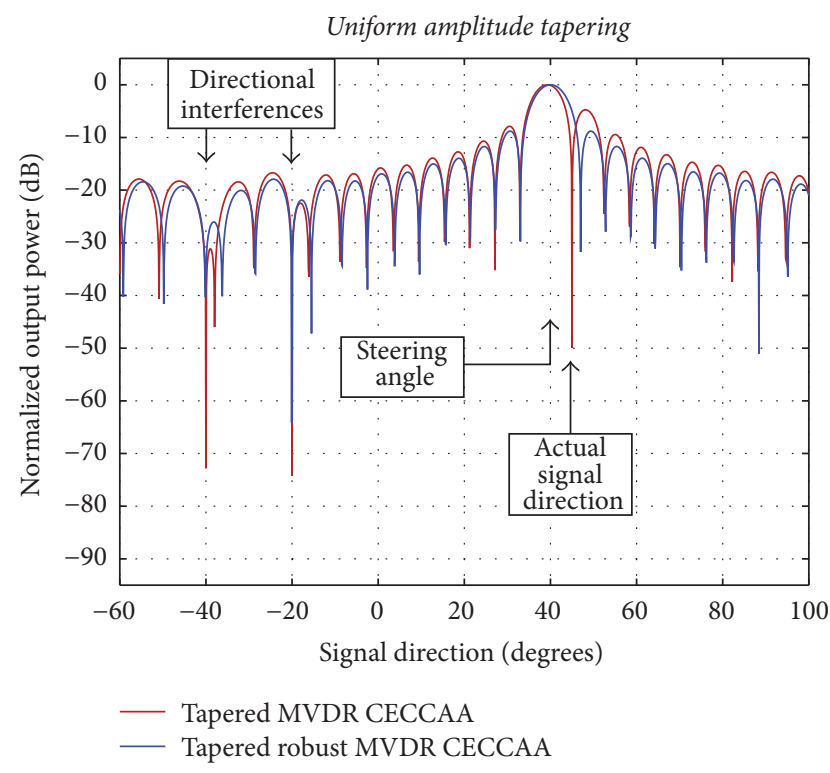

(a)

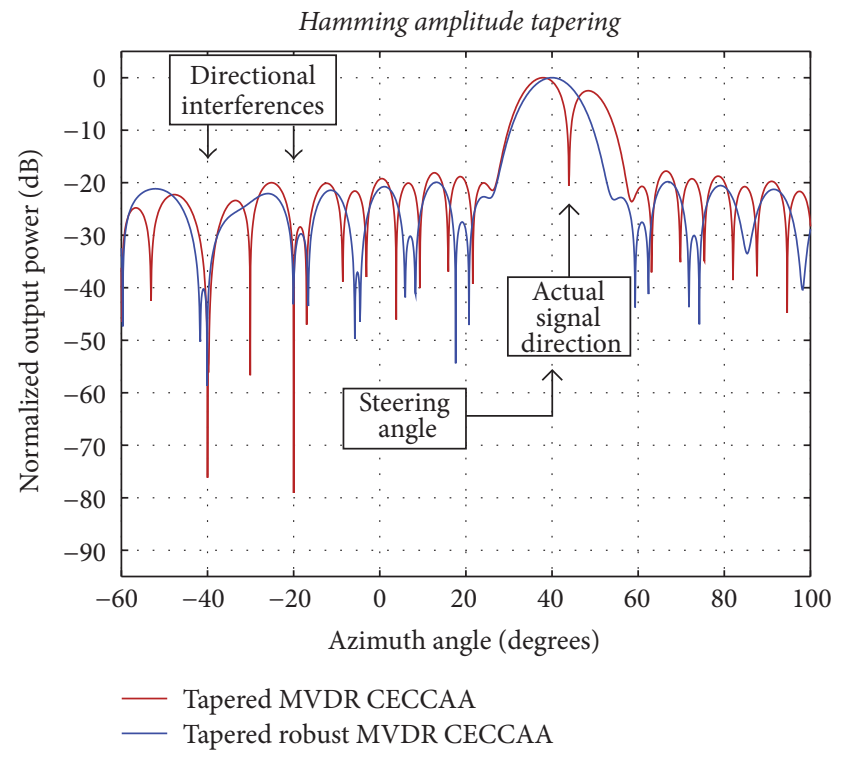

(b)

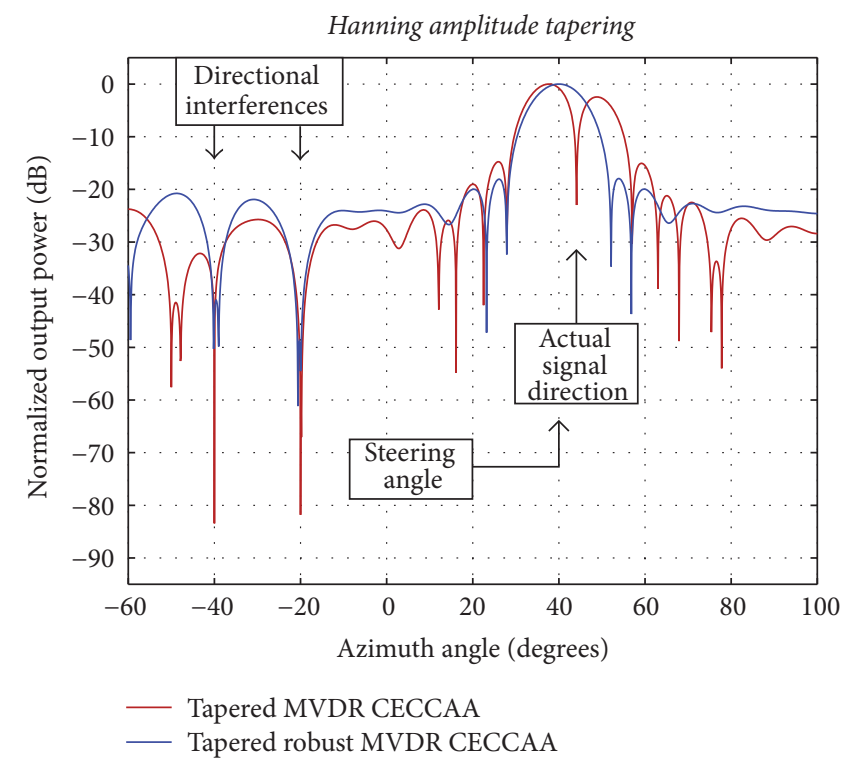

(c)

FIGURE 8: (a) Normalized power pattern comparison of the MVDR beamformer and robust MVDR beamformer by using uniform tapering in the presence of array imperfections. (b) Normalized power pattern comparison of the tapered MVDR beamformer and proposed tapered robust MVDR beamformer by using Hamming amplitude tapering in the presence of array imperfections. (c) Normalized power pattern comparison of the tapered MVDR beamformer and proposed tapered robust MVDR beamformer by using Hanning amplitude tapering in the presence of array imperfections.

with an element in the center by using different tapering windows with the variation of azimuth angle and elevation angle. To sketch those figures, actual and assumed signal directions are both considered at $0^{\circ}$. It is observed that the proposed beamformer by using Triangular, Hamming, and Hanning amplitude tapering exhibits low SLL in comparison to beamformer using uniform amplitude tapering.

Figure 10 depicts the normalized power pattern comparison of the proposed CECCAA with the conventional
CECCAA beamformer. From this figure, it is observed that the proposed beamformer not only is able to attenuate the directional interferences but also is able to reduce the side lobe level. Moreover, the proposed beamformer is robust against array imperfections. It is observed from Figures 8 and 10 that the normalized output power for conventional beamformer in the presence of array imperfections without using any robust technique is $-49.9 \mathrm{~dB}$, whereas the normalized output powers of the proposed CECCAA beamformer 


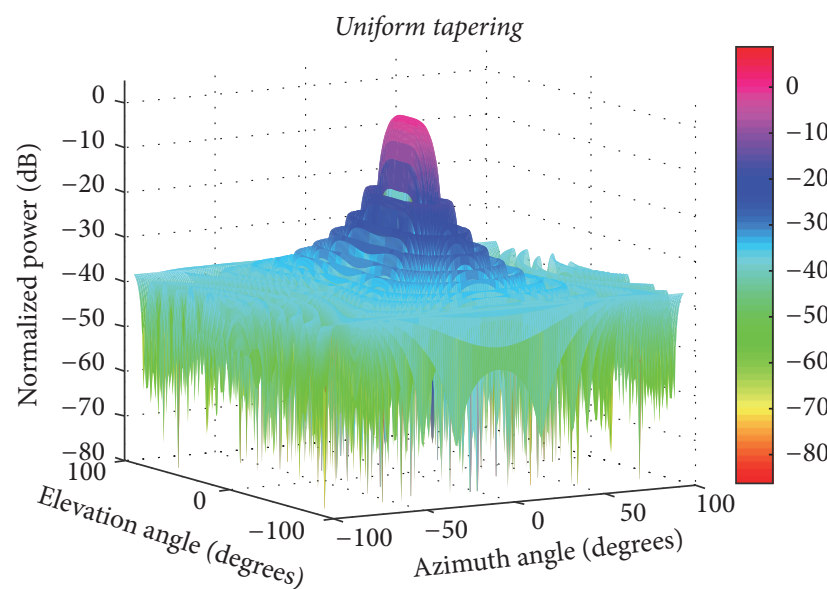

(a)

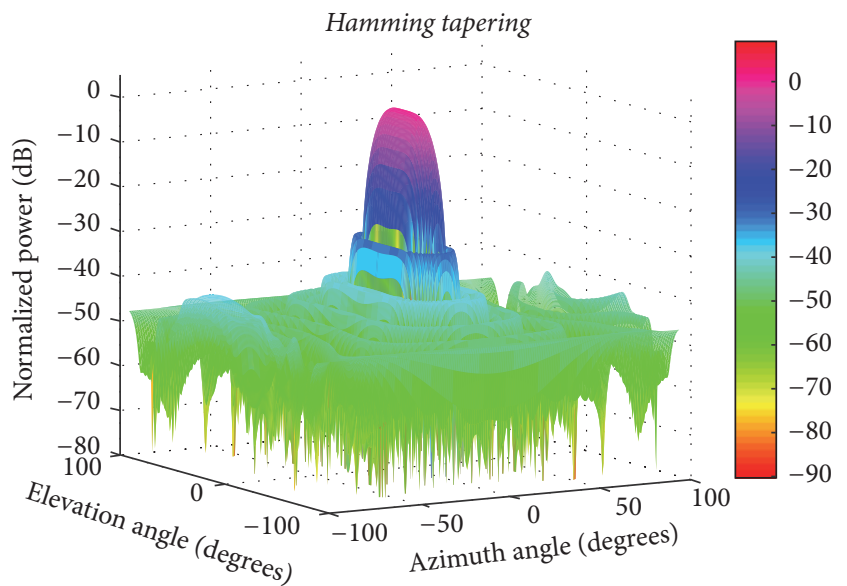

(c)

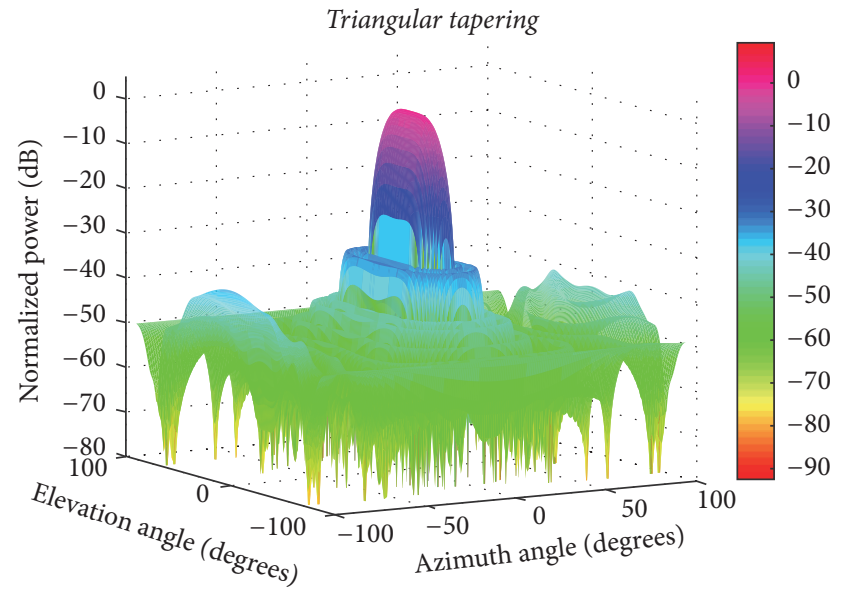

(b)

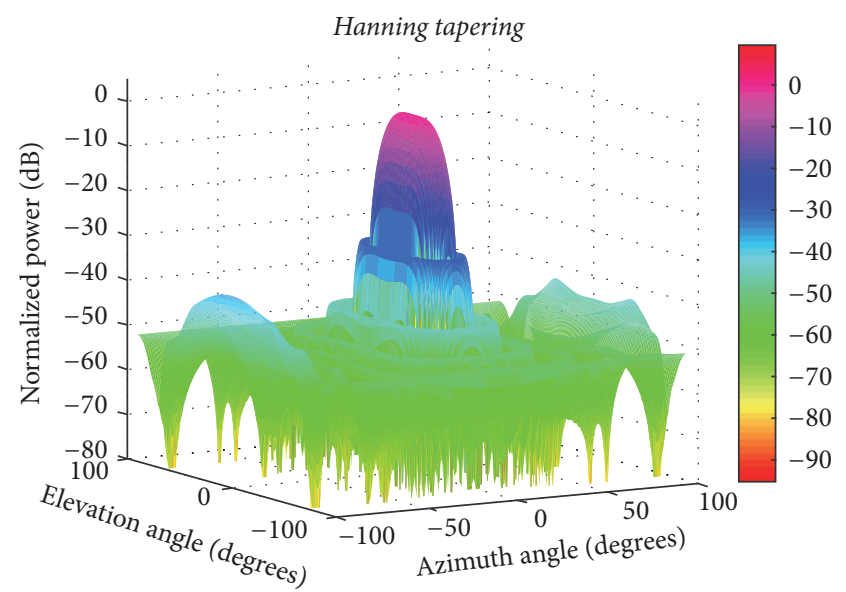

(d)

FIGURE 9: Normalized 3D output power patterns of (a) uniform tapered, (b) Triangular tapered, (c) Hamming tapered, and (d) Hanning tapered MVDR CECCAA beamformers with the variation of azimuth angle and elevation angle.

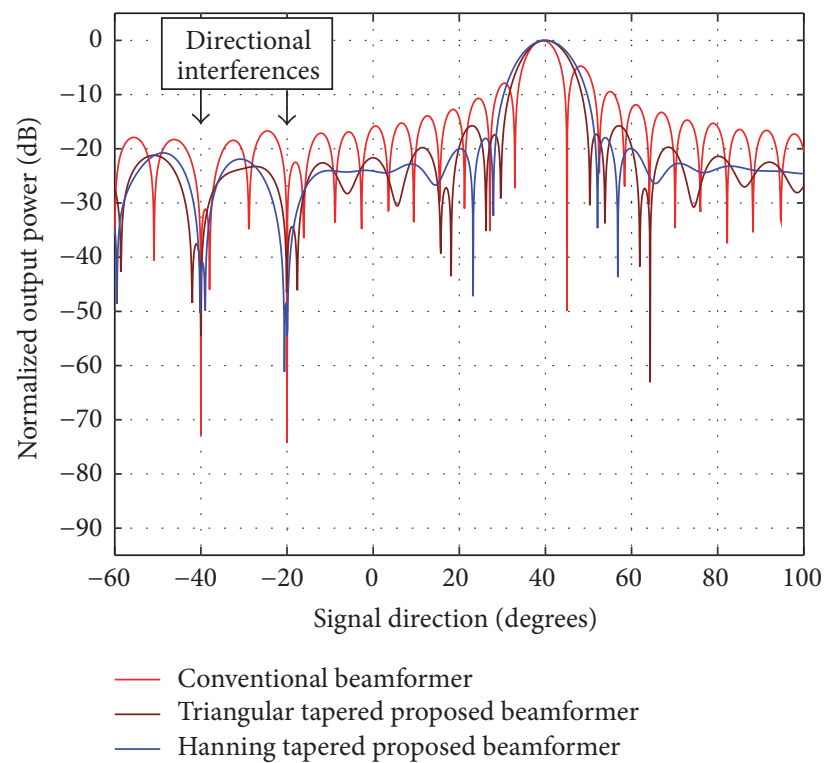

FIGURE 10: Normalized power pattern comparison of the proposed CECCAA beamformer with the conventional CECCAA beamformer. 
are increased to $-2.865 \mathrm{~dB},-2.395 \mathrm{~dB}$, and $-2.195 \mathrm{~dB}$ by using Triangular, Hamming, and Hanning tapering, respectively. Also, the SLL of the robust MVDR beamformer without using tapering is $-8.88 \mathrm{~dB}$. The SLL of the proposed beamformer reduced to $-17.35 \mathrm{~dB},-19.91 \mathrm{~dB}$, and $-17.95 \mathrm{~dB}$ using Triangular, Hamming, and Hanning tapering, respectively.

\section{Conclusion}

Tapered robust CECCAA beamformer by using different tapering windows and VDL technique has been presented in this paper. The simulation results have demonstrated that the proposed beamformer is able to (a) reduce the SLL, (b) attenuate the directional interfering signals, and (c) improve the output signal power and signal-to-noise ratio in the presence of signal direction mismatch. It has been observed that normalized output of the proposed beamformer using Hanning tapering has increased by $47.705 \mathrm{~dB}$ and SLL has reduced by $9.07 \mathrm{~dB}$ in comparison to conventional CECCAA beamformer. The numerical examples have also established that the proposed CECCAA structure offers lower SLL in comparison to a CCAA structure.

\section{Conflicts of Interest}

The authors declare that there are no conflicts of interest regarding the publication of this article.

\section{References}

[1] L. C. Godara, Smart Antenna, CRC Press, 2004.

[2] C. A. Balanis, Antenna Theory: Analysis and Design, John Wiley and Sons, New York, NY, USA, 2005.

[3] M. S. Hossain, M. F. Reza, M. M. Rashid, and M. F. Ali, "Performance analysis of broadband concentric circular antenna array processor," in Proceedings of the 5th International Conference on Informatics, Electronics and Vision, ICIEV 2016, pp. 1047-1051, bgd, May 2016.

[4] G. Ram, D. Mandal, R. Kar, and S. P. Ghoshal, "Cat swarm optimization as applied to time-modulated concentric circular antenna array: analysis and comparison with other stochastic optimization methods," IEEE Transactions on Antennas and Propagation, vol. 63, no. 9, pp. 4180-4183, 2015.

[5] X. Zhao, Q. Yang, and Y. Zhang, "Application of TLBO to synthesis of sparse concentric ring arrays," in Proceedings of the 10th European Conference on Antennas and Propagation, EuCAP 2016, Davos, Switzerland, April 2016.

[6] M. Nofal, S. Aljahdali, and Y. Albagory, “Tapered beamforming for concentric ring arrays," AEU - International Journal of Electronics and Communications, vol. 67, no. 1, pp. 58-63, 2013.

[7] R. L. Haupt, "Optimized element spacing for low sidelobe concentric ring arrays," IEEE Transactions on Antennas and Propagation, vol. 56, no. 1, pp. 266-268, 2008.

[8] X. Zhao, Q. Yang, and Y. Zhang, "A hybrid method for the optimal synthesis of 3-D patterns of sparse concentric ring arrays," Institute of Electrical and Electronics Engineers. Transactions on Antennas and Propagation, vol. 64, no. 2, pp. 515-524, 2016.

[9] N. Yuri and P. Ilia, "Probability of false peaks occuring via circular and concentric antenna arrays DOA estimation," in
Proceedings of the 39th International Conference on Telecommunications and Signal Processing, TSP 2016, pp. 178-181, aut, June 2016.

[10] R. Goossens and H. Rogier, "Direction-of-arrival and polarization estimation with uniform circular arrays in the presence of mutual coupling," AEU-International Journal of Electronics and Communications, vol. 62, no. 3, pp. 199-206, 2008.

[11] M. I. Dessouky, H. A. Sharshar, and Y. A. Albagory, "Efficient sidelobe reduction technique for small-sized concentric circular arrays," Progress in Electromagnetics Research, vol. 65, pp. 187200, 2006.

[12] M. Dessouky, H. Sharshar, and Y. Albagory, "A novel tapered beamforming window for uniform concentric circular arrays," Journal of Electromagnetic Waves and Applications, vol. 20, no. 14, pp. 2077-2089, 2006.

[13] M. F. Reza, M. S. Hossain, and M. M. Rashid, "Robust uniform concentric circular array beamforming in the existence of look direction disparity," in Proceedings of the 2nd International Conference on Electrical, Computer and Telecommunication Engineering (ICECTE), p. 1-4, 8-10, Rajshahi, Bangladesh, December 2016.

[14] S. A. Vorobyov, A. B. Gershman, and Z.-Q. Luo, "Robust adaptive beamforming using worst-case performance optimization: a solution to the signal mismatch problem," IEEE Transactions on Signal Processing, vol. 51, no. 2, pp. 313-324, 2003.

[15] X. L. Yuan, L. Gan, and H. S. Liao, "A robust interference covariance matrix reconstruction algorithm against arbitrary interference steering vector mismatch," IEICE Transactions on Fundamentals of Electronics, Communications and Computer Sciences, vol. E98A, no. 7, pp. 1553-1557, 2015.

[16] O. Besson, A. A. Monakov, and C. Chalus, "Signal waveform estimation in the presence of uncertainties about the steering vector," IEEE Transactions on Signal Processing, vol. 52, no. 9, pp. 2432-2440, 2004.

[17] T. Zhang and L. Sun, "Iterative robust capon beamforming with adaptively updated array steering vector mismatch levels," International Scholarly Research Notices, vol. 2014, Article ID 260875, pp. 1-8, 2014.

[18] W. Li, X. Mao, Z. Zhai, and Y. Li, "High performance robust adaptive beamforming in the presence of array imperfections," International Journal of Antennas and Propagation, vol. 2016, Article ID 3743509, 2016.

[19] M. S. Hossain, L. C. Godara, and M. R. Islam, "Efficient robust broadband beamforming algorithms using variable loading," IEEE Latin America Transactions, vol. 10, no. 3, pp. 1697-1702, 2012.

[20] M. S. Hossain, G. N. Milford, M. C. Reed, and L. C. Godara, "Efficient robust broadband antenna array processor in the presence of look direction errors," Institute of Electrical and Electronics Engineers. Transactions on Antennas and Propagation, vol. 61, no. 2, pp. 718-727, 2013.

[21] J. Li, P. Stoica, and Z. Wang, "On robust Capon beamforming and diagonal loading," IEEE Transactions on Signal Processing, vol. 51, no. 7, pp. 1702-1715, 2003.

[22] M. F. Reza and M. S. Hossain, "Robust concentric circular antenna array with variable loading technique in the presence of look direction disparity," Progress In Electromagnetics Research M, vol. 57, pp. 35-43, 2017.

[23] W. Wang, R. Wu, and J. Liang, "A novel diagonal loading method for robust adaptive beamforming," Progress In Electromagnetics Research C, vol. 18, pp. 245-255, 2011. 
[24] L. Gan and Z. Yi, "Automatic computation of diagonal loading factor for robust adaptive beamforming based on Gaussian distribution," AEU - International Journal of Electronics and Communications, vol. 67, no. 7, pp. 570-573, 2013.

[25] H. Chen, Q. Wan, X. Zhang, and R. Fan, "Robust beamforming with inter-atom-interference mitigation approach for uniform circular arrays," AEU - International Journal of Electronics and Communications, vol. 69, no. 1, pp. 236-241, 2014. 


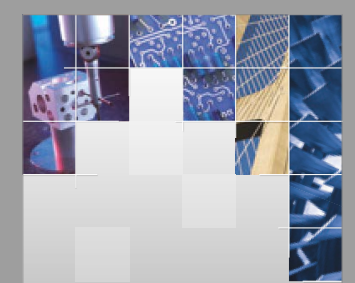

\section{Enfincering}
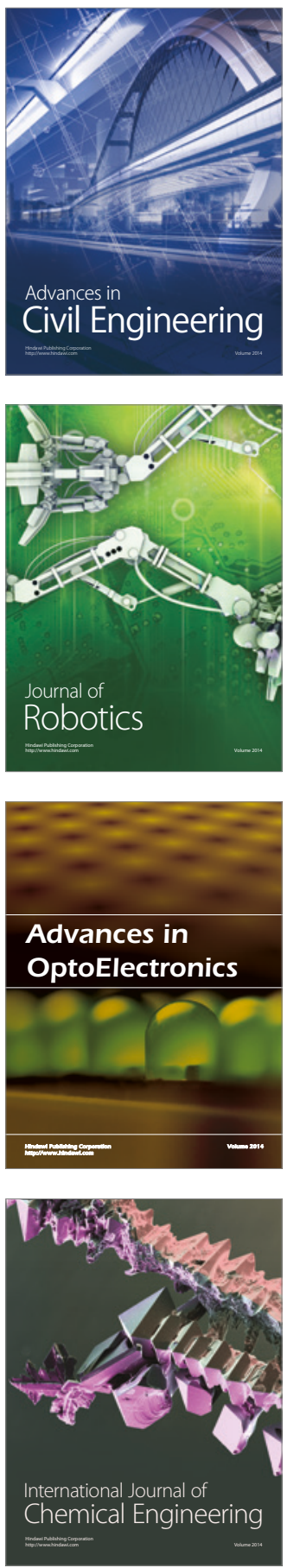

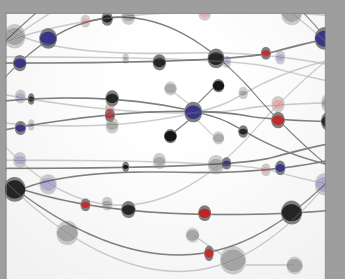

The Scientific World Journal

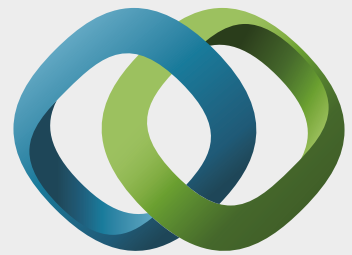

\section{Hindawi}

Submit your manuscripts at

https://www.hindawi.com
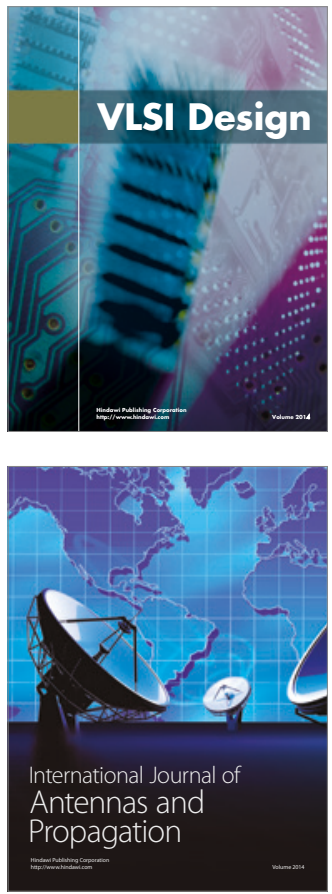

\section{Rotating}

Machinery
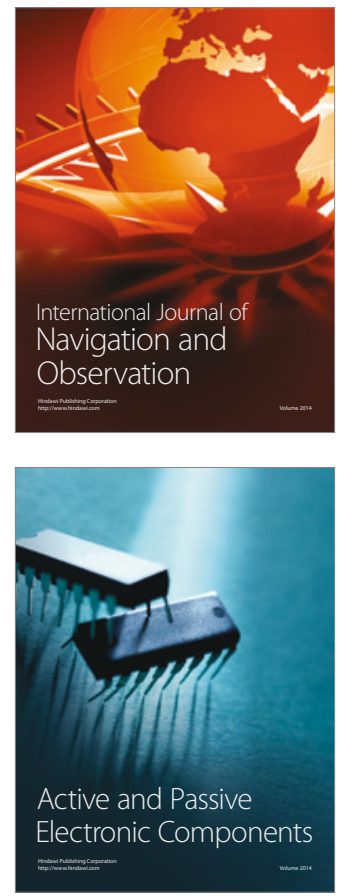
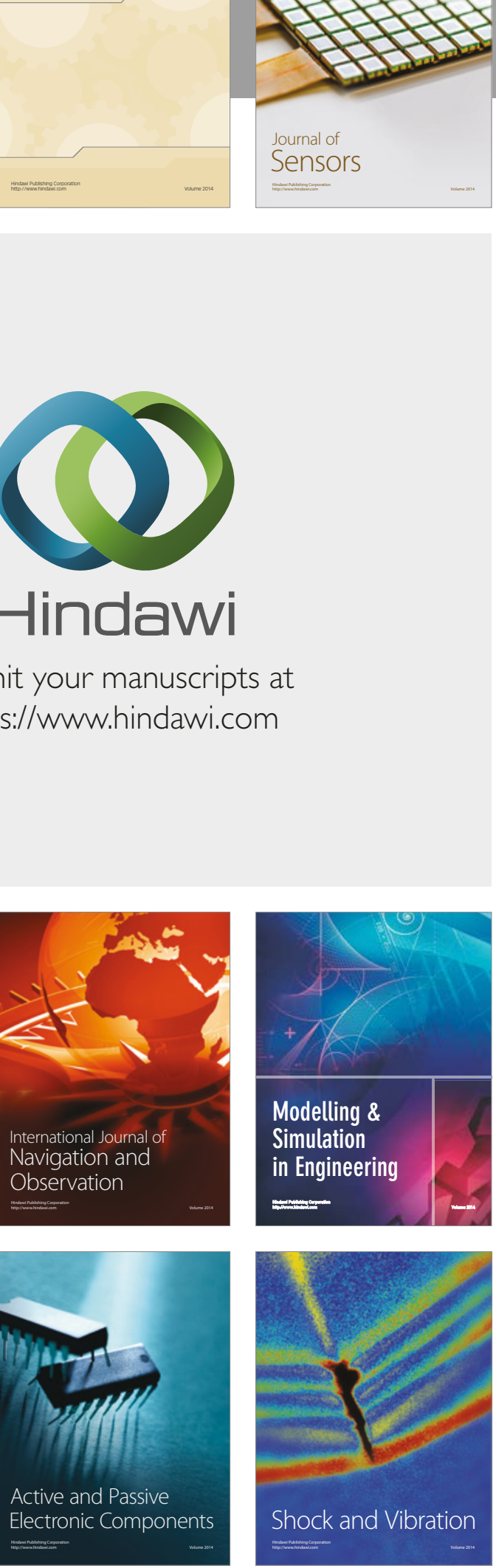
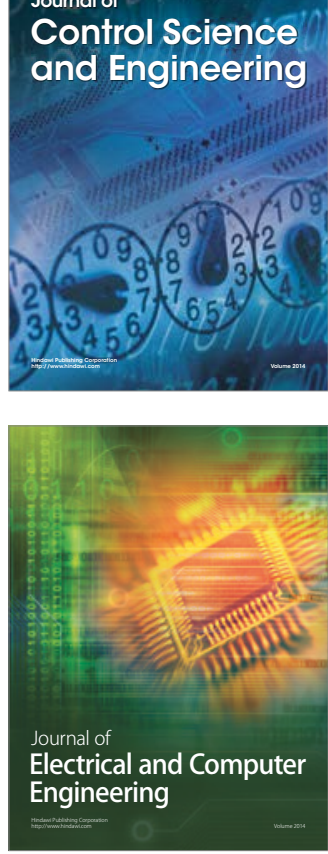

Distributed

Journal of

Control Science

and Engineering
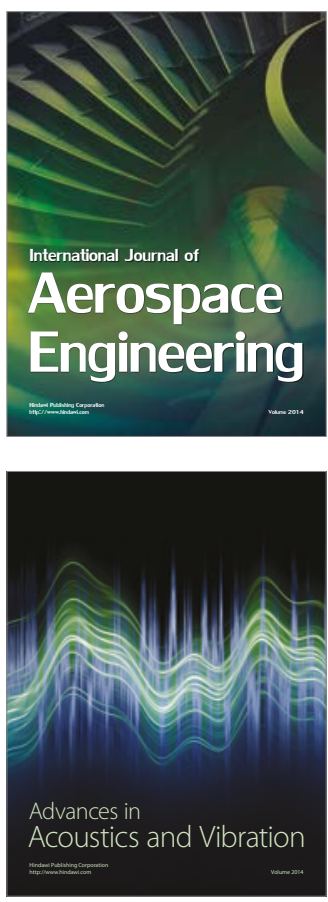

Sensor Networks 